\title{
EL NACIMIENTO DE UNA UNIVERSIDAD EN EL VALLE CENTRAL Y LA AMPUTACIÓN DE LAS SEDES REGIONALES DE LA UNIVERSIDAD DE CHILE: EL CASO DE LA UNIVERSIDAD DE TALCA
}

Francisco Javier Pinedo Castro 


\section{FRANCISCO JAVIER PINEDO CASTRO}

Licenciado en Literatura. Doctor en Filología Románica, Universidad Católica de Lovaina, Bélgica. Profesor titular de la Universidad de Talca, director del Instituto de Estudios Humanísticos Abate Molina y director de la Revista de Humanidades y Ciencias Sociales, Universum. Áreas de interés: Historia de las ideas y literatura en Chile y América Latina. 


\section{EL NACIMIENTO DE UNA UNIVERSIDAD EN EL VALLE CENTRAL Y LA AMPUTACIÓN DE LAS SEDES REGIONALES DE LA UNIVERSIDAD DE CHILE: EL CASO DE LA UNIVERSIDAD DE TALCA}

\section{INTRODUCCIÓN}

La fundación de sedes en algunas regiones del país para cultivar estudios superiores y, particularmente, en el caso de Talca, tiene diversas fuentes que fueron organizándose para alcanzar ese propósito, con iniciativas tanto desde la propia ciudad como desde el poder político central.

Entre estas se pueden nombrar las acciones de personalidades como Irma Salas y los rectores de la Universidad de Chile y Universidad Técnica del Estado, Juan Gómez Millas y Horacio Aravena Andaur, al presidente Frei Montalva y el Plan California, a la presión de la comunidad regional de Talca, a ciertos efectos de la reforma universitaria de 1967 y 1968², entre otros, todos ellos zurcidos en los creativos y convulsos años 60 del siglo XX, a lo que se debe agregar las consecuencias de la reforma de 1981 impuesta por el Gobierno militar.

\section{PRIMEROS ANTECEDENTES}

Antes, todavía, se deben mencionar algunos antecedentes de esta historia en la cual todos los argumentos y circunstancias que permitieran alcanzar el objetivo de tener una Universidad regional fueron considerados válidos para lograr este propósito. Se menciona, por ejemplo, los esfuerzos que durante el siglo XIX se realizaron con la implementación del Decreto 301 de abril de 1855, que estableció que el Director de la Escuela de Artes y Oficios de Santiago se trasladase a Talca para preparar un local en el cual funcionaría una escuela similar en esta ciudad. Una iniciativa que no prosperó.

Talca era una ciudad que desde mediados de aquel siglo había alcanzado cierto desarrollo agrícola e industrial, lo que había permitido el surgimiento de un sector social interesado en la cultura, la política y las artes a nivel nacional y a veces internacional, con figuras como Carmen Arriagada, los hermanos Gana López, Carmen Cruzat, Juan de la Cruz Donoso Cienfuegos, Vicente Antúnez, y su entorno de artistas y pensadores, entre los cuales el pintor bávaro Mauricio Rugendas brillaba en la escasa luz de una provincia que hacía esfuerzos para conocer lo que sucedía en el mundo². En 1844 se

1. Véase Brunner, J.J., Educación superior en Chile, Instituciones, Mercados y Políticas Gubernamentales (19672007), Santiago: Ediciones UDP, 2009.

2. Véase Pinochet de la Barra, O., "Carmen Arriagada, algunos rasgos humanos", en Revista Universum, Universidad de Talca, № 16, 2001. 
funda el periódico El Alfa, el primero en la zona centro-sur del país (dirigido justamente por Carmen Arriagada) que recogía artículos sobre cultura y política, comentarios y opiniones del grupo ilustrado regional; más tarde, en 1875 se inauguró el Teatro Municipal que contribuyó a la difusión del arte escénico, y en septiembre de ese mismo año llegó el ferrocarril a la ciudad, todo lo cual permitió el desarrollo de cierta cultura que iba manifestando la necesidad de poseer centros de formación y reflexión universitaria, que resultaban ya antiguos en Europa e incluso en otros lugares de América, pero que en el caso nuestro se iban postergando por la siempre urgente necesidad de resolver insuficiencias económicas ${ }^{3}$.

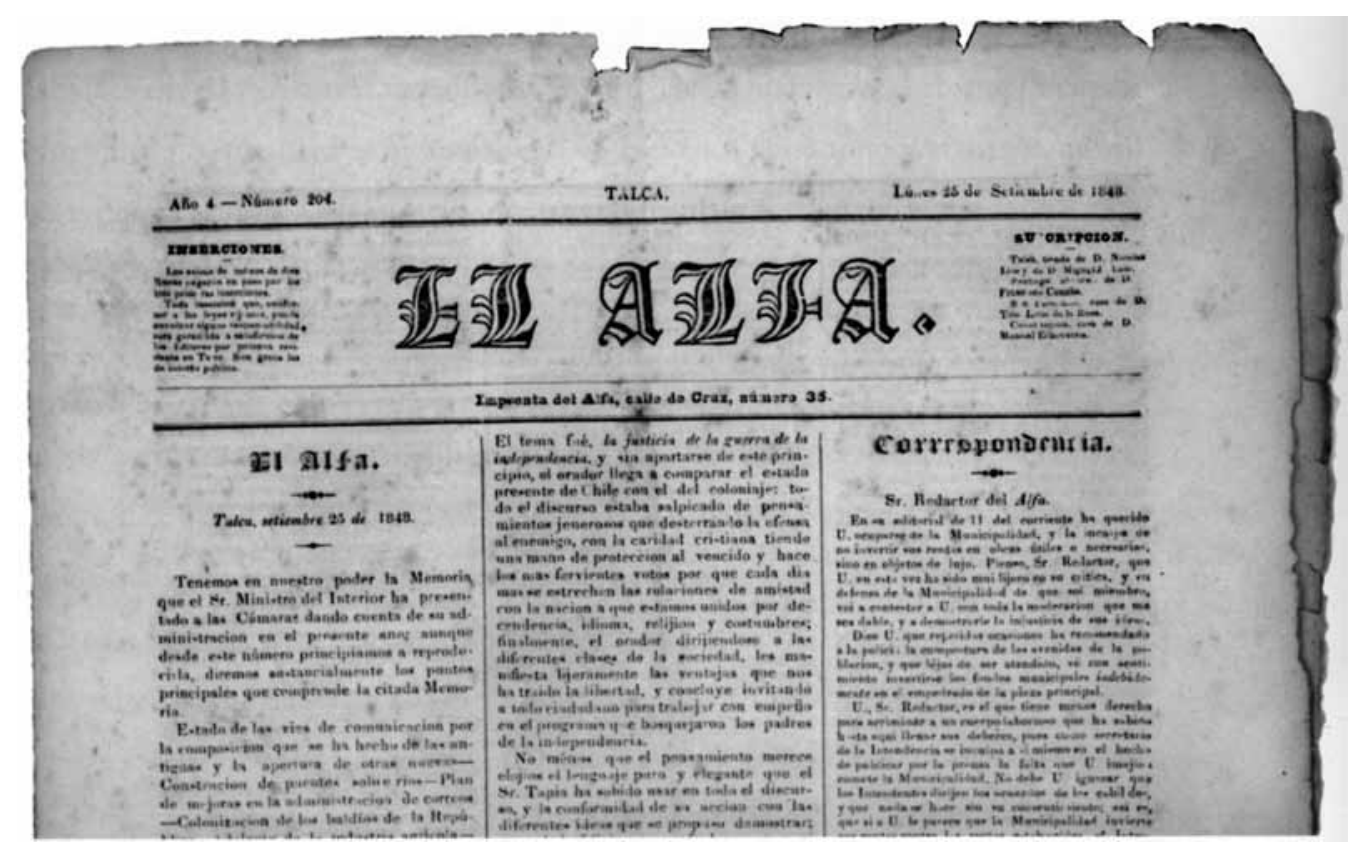

Diario El Alfa, creado en 1844, por Carmen Arriagada.

Todavía, durante la post Independencia, el año 1827 se había fundado el Instituto Literario, hoy Liceo Abate Juan Ignacio Molina, con los fondos entregados por el ilustre jesuita maulino en su testamento ${ }^{4}$ para que en Talca se creara un centro de educación para

3. Véase Pinedo, J., "Identidad en la región del Maule. Reflexiones e imágenes sobre el tema", en Revista Universum, Universidad de Talca, Año 14, 1999, págs. 151-180.

4. Fue durante el viaje del Obispo Cienfuegos a Bolonia en 1823, como representante de Chile ante la Santa Sede, que Juan Ignacio Molina le hizo donación de toda su herencia con el objetivo de fundar en Talca "una biblioteca pública de libros de ciencias y artes útiles y provista de instrumentos de física, aptos para el estudio de la astronomía, náutica y matemáticas". Véase Briones Toledo, H., El Abate Juan Ignacio Molina. Ensayo crítico - introductorio a su vida y obra, Santiago: Andrés Bello, 1968, pág. 158. 
jóvenes de la región, siguiendo el lema de la Universidad de Bolonia: "Bolonia docet", que Molina intentó replicar en su ciudad: "Talca, docet", Talca enseñas.

Juan Ignacio Molina, nacido en la ribera sur del río Maule, en la hacienda Huaraculén en 1740 y fallecido en Bolonia, Italia, en 1829, fue un hombre sabio que incorporó la naturaleza y los habitantes de Chile a la ciencia universal, y para quien la lectura, la observación y la experimentación resultaban fundamentales en su ilustrada mentalidad.

Y aun se mencionan dos antecedentes más: se dice que a mediados del siglo XX don Eduardo Donoso Cruz, un conocido agricultor de la zona, habría dejado una parte de su patrimonio con el propósito de fundar una Universidad en Talca, lo que nunca se llevó a cabo.

Y, a comienzos del siglo XX, don Enrique Molina Garmendia, rector del Liceo de Hombres de Talca entre 1905 y 1915, acompañado de un grupo de intelectuales y profesores del Liceo, muchos de ellos pertenecientes a la masonería, intentaron levantar una Universidad, pero esta se vio impedida por la presión de grupos católicos conservadores que temieron que desde esa institución se pudiera afectar la conciencia de las jóvenes generaciones. El fracaso de este proyecto obligó al rector Molina a emigrar a la ciudad de Concepción, donde fue bien recibido (por los masones) quienes contribuyeron a fundar la Universidad de Concepción en 1919, siendo Enrique Molina su rector hasta 1956.

Talca perdió la oportunidad de poseer una Casa de Estudios superiores en las primeras décadas del siglo XX, lo que probablemente habría evitado la larga crisis económica y cultural que se instaló en la ciudad hasta bien entrado el siglo XX por ausencia de líderes que hubieran desarrollado la región con propuestas innovadoras, confirmado la idea de que entre las ciudades con y sin universidad existen profundas diferencias de desarrollo económico, capital humano y calidad de vida, a favor de las primeras.

Volviendo a los años 50 y 60, observamos una serie de iniciativas llevadas a efecto desde el Gobierno central como desde la comunidad local para lograr el propósito mencionado.

Por el lado de la Universidad de Chile, desde finales de la década del 50 se pensó fundar sedes en regiones, denominadas con el nombre genérico de "Colegios universitarios" o "Sedes regionales", y se reconoce los esfuerzos de Juan Gómez Millas, quien fue rector de la Universidad de Chile durante diez años (entre 1953 y 1963) y más tarde, ministro de Educación (entre 1964 y 1968), durante la presidencia de Eduardo Frei Montalva. Anteriormente, en 1952, había sido nombrado ministro de Educación Pública por el presidente Ibáñez, cargo en el que estuvo un año antes de ser elegido rector de la Universidad de Chile ${ }^{6}$.

5. Walter Hanisch en muchas de sus obras ha expuesto la intención de Molina para alcanzar este propósito. Véase Pinedo, J., "El más universal de los maulinos", en Walter Hanisch, Juan I. Molina y sus obras, Universidad de Talca, 1999.

6. Existe una amplia bibliografía sobre Gómez Millas y sobre la reforma universitaria. Véase, entre otros, http: //es.goldenmap.com/Juan_G\%C3\%B3mez_Millas 
En 1954, estimulados por un grupo de profesionales egresados de la Universidad de Chile, se creó en Talca una filial; en su inauguración el rector Juan Gómez Millas hizo suyo el interés de la ciudad por vincularse con la Universidad nacional.

Tanto el rector de la Universidad de Chile, Juan Gómez Millas, como el rector de la Universidad Técnica, Horacio Aravena Andaur, comprendieron esta solicitud y dieron luz verde a una serie de acciones que permitieran converger en una Universidad.

Algo más tarde, en 1957, se realizó un Seminario de Estudios Regionales en Talca, se presentó un trabajo titulado "Realidad Educacional" en el que se analizó, a la luz de numerosos antecedentes, la situación económica de la región y, particularmente, la relación del desarrollo económico con el problema educacional.

En la misma sesión plenaria se propuso la designación de una comisión que quedó integrada por: el Dr. César Caravagno, Samuel Jiménez Sepúlveda, Hernán Cruz Concha, Marcelo Cruz Fuenzalida y los directivos de los colegios de enseñanza media. La comisión realizó diversas gestiones y a su trabajo se sumaron, posteriormente, distintas personalidades: Jorge Plaza Cruz, en representación de la comunidad PlazaTejada, quien además ofreció una importante donación de siete hectáreas de terreno. Alfonso Fernández, presidente del Centro para el Progreso, Juan C. Bravo, Miguel Ángel Escobar, y Lautaro Mardones, entre otros, quienes efectuaron las diligencias tendientes a lograr que la Universidad Técnica del Estado creara una sede en Talca. Su rector, Horacio Aravena Andaur y el Honorable Consejo Universitario, en sesión del 28 de octubre de 1960, autorizaron la creación de cursos universitarios en Talca ${ }^{7}$.

\section{LOS AÑOS 60}

Este programa de fundación de colegios universitarios fue implementado finalmente por la profesora Irma Salas Silva (1903-1987), una destacada educadora, hija de Darío Salas, que fue la primera chilena en obtener un doctorado en Educación, en la Universidad de Columbia, EE.UU., en $1930^{8}$.

Irma Salas fue nombrada "Directora General de los Colegios Universitarios (19601964), creados en 1960 bajo la rectoría de don Juan Gómez Millas con el fin de extenderse a las provincias y crear allí carreras cortas, descentralizando a la Universidad; de esta forma, surgieron las Sedes de Temuco (13/07/1960), La Serena (1/11/1960), Antofagasta, Talca y Osorno (13/12/1962). En 1965 se transformaron en los Centros Universitarios de Provincias, siendo su primera Coordinadora la propia Irma Salas entre 1965 y 1966"9.

7. AAVV., Universidad de Talca. Veinte Años de Historia, Talca, 2001.

8. Irma Salas participó en la creación del Liceo Experimental Manuel de Salas, donde fue Directora hasta 1943. Este fue un centro de experimentación docente perteneciente al Instituto Pedagógico, en el que durante años se puso en práctica la pedagogía de John Dewey. Véase Caiceo Escudero, J., "El Instituto Pedagógico y el sistema educacional chileno", en Revista Intramuros, №10, diciembre de 2002, págs. 33-8.

9. Caiceo Escudero, J., "Amanda Labarca, Irma Salas y Mabel Condemarín, Tres educadoras laicas y feministas del siglo XX en Chile”, Rev. Teoria e Prática da Educação, v. 13, N 1, jan./abr. 2010, págs. 105-116. 


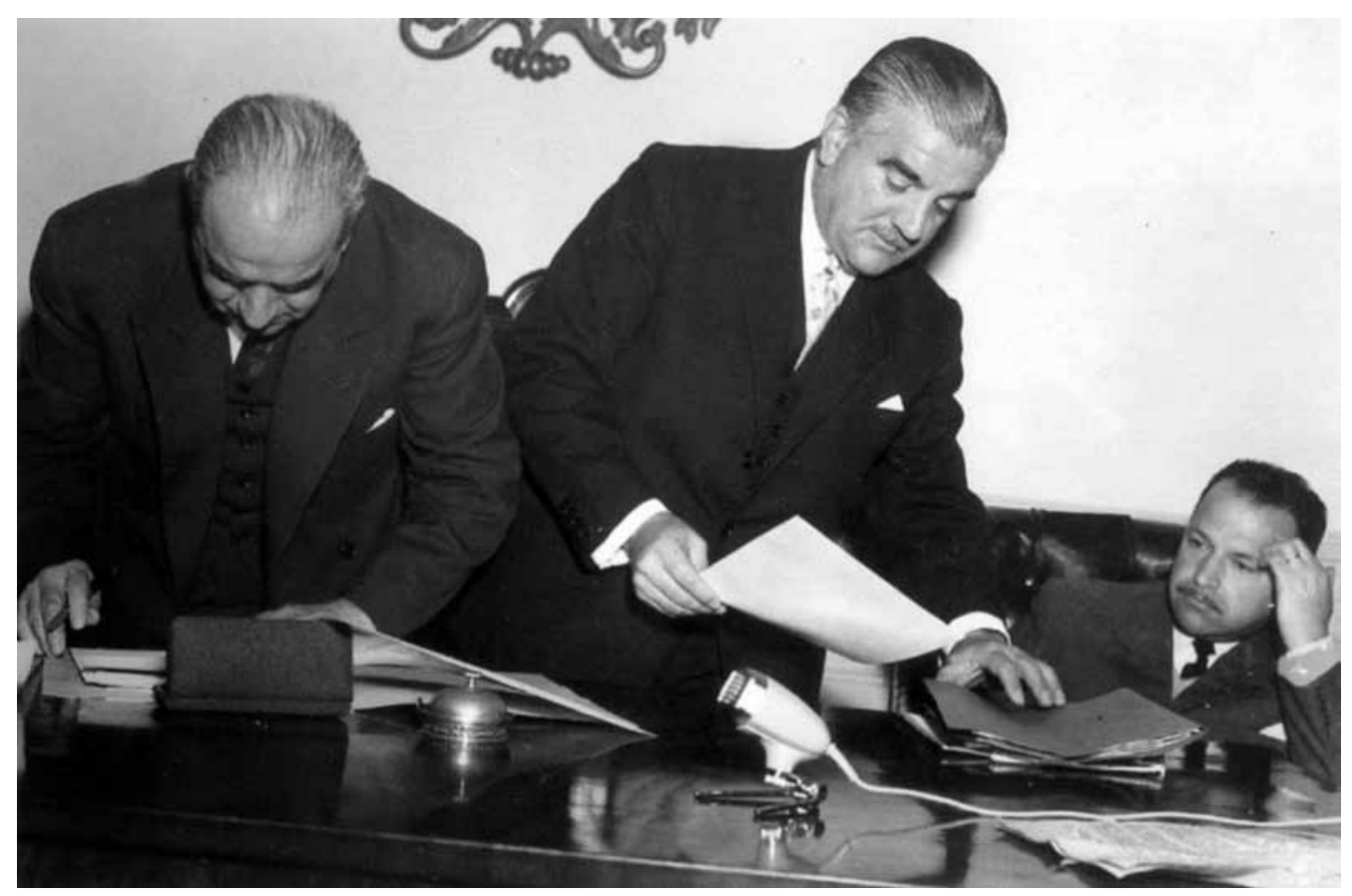

Al centro el alcalde Galo Lavín; a su derecha, el rector Juan Gómez Millas; a su izquierda, Enrique Sepúlveda, Secretario Municipal. Tres grandes promotores de una sede de la Universidad de Chile en Talca.

\section{EL PLAN CALIFORNIA}

El Estado de California, cuyo funcionamiento Irma Salas probablemente conoció en su estancia norteamericana, reconoce entre tres tipos de instituciones de educación superior: las "Universities of California", dedicadas a la investigación; las "California State Universities", sin investigación, y los "Community Colleges", con funciones de docencia específica. En este sistema tripartito los estudiantes tienen reservados ingresos según sus rendimientos escolares, aunque existen las posibilidades de movilidad interna en la medida en que el estudiante va superando etapas.

Con algunas ideas que venían desde fines del Gobierno de Jorge Alessandri (19581964), al llegar al Gobierno de Eduardo Frei Montalva (1964-1970) se permitió extender la Universidad de Chile a nueve regiones del país: las sedes de Arica, Iquique, Antofagasta, La Serena, Valparaíso, Talca, Nuble, Temuco y Osorno. Este presidente fue un hombre progresista con un programa progresista, que puso en práctica un intento por descentralizar y modernizar al país, y redefinir el rol del Estado y, particularmente, de la educación. La creación de centros regionales de educación superior coincidía plenamente con las propuestas de Irma Salas. 
Como consecuencia de estos vínculos fue que en la década de los 60 se lanzó, dentro del contexto de la Alianza para el Progreso impulsada por el Gobierno de los Estados Unidos de Norteamérica y bajo las administraciones de los Presidentes John F. Kennedy y Eduardo Frei Montalva, el primer Plan Chile-California, denominado "Ayuda para el Desarrollo", estableciéndose cooperación técnica en áreas tan variadas como la agricultura, educación, gestión de recursos hídricos y transportes ${ }^{10}$.

El plan fue concebido como una herramienta de desarrollo y superación de la pobreza y la marginalidad social, una herramienta fundamental que a través de programas específicos permitieran ampliar la calidad profesional de los habitantes de las regiones.

Oficialmente iniciado en el año 1963 por el entonces Gobernador del Estado de California, señor Pat Brown, y presidido en Chile por el entonces Director de Presupuestos del Ministerio de Hacienda, señor Edgardo Boeninger, el primer Plan buscaba desarrollar a nuestro país en ámbitos claves para su progreso como Nación, según las necesidades propias de un país subdesarrollado. La implementación del Plan permitió el inicio del denominado "Convenio", entre el Sistema de Universidades de California y la Universidad de Chile, en el año 1965. Este convenio fue financiado por la Fundación Ford y permitió un nutrido intercambio académico entre ambas casas de estudio, a través del envío de profesores a la Universidad de Chile y el de alumnos a la Universidad de Davis.

Además de los proyectos de la Universidad de Andrés Bello, la Universidad Técnica del Estado tenía sus propios planes para desarrollar actividades en la región del Maule y en otras del país.

Así, el 17 de octubre de 1962 fue creado el primer centro universitario en Talca dependiente de la Universidad Técnica del Estado. Uno de los testigos de esta historia, don Lautaro Mardones, en una entrevista en octubre de 2011 en el Diario La Ciudad, señala esta voluntad y el perfil técnico e industrial de la nueva institución: "El Centro Universitario de 1962 fue creado precisamente para satisfacer las necesidades de enseñanza de la zona y esas demandas eran industriales, técnicas".

Una historia que hoy se narra con sentido épico: el profesor Mardones nos cuenta que viajaron a Santiago en el auto del alcalde Galo Lavín a entrevistarse con su amigo el rector de la Universidad Técnica, Horacio Aravena Andaur. Sin embargo, a la salida de San Bernardo se quedaron en pana. Y era tal el entusiasmo y la convicción, que tomaron una carreta y llegaron a la reunión con el rector: “„En carreta de feria!”. La anécdota sirve para entender las dificultades del origen, pero "por sobre todo las ganas de hacer las cosas". "Hay que tener ganas", concluye Mardones.

10. Véase http: //spanish.chile.usembassy.gov/root/pdfs/resumen-ejec-plan-chile--calif0409.pdf 


\section{LAS ACCIONES SE VAN SUMANDO}

El senador Ulises Correa (del Partido Radical) y el diputado René Lagos (diputado del Partido Radical entre 1961-1965, y masón) realizaron gestiones para concretar la donación de una casona ubicada en la calle 4 oriente entre la 1 y la 2 norte que pertenecía a la colonia italiana a través de don Juan Bertucci, quien acogió la demanda de los parlamentarios para que en ella se dictaran los primeros cursos de la sede de la Universidad Técnica. La entrega se realizó el 26 de abril de 1962, con presencia del alcalde de Talca, Galo Lavín Pradenas (alcalde entre 1961-1963). El anuncio oficial de creación del Centro Universitario Universidad Técnica del Estado se llevó a cabo el 14 de noviembre de 1962, momento en que se nombró Director del Centro a don Samuel Jiménez Sepúlveda.

\section{LOS ESFUERZOS DE LA COMUNIDAD DE TALCA}

Por las razones anteriores, la actual Universidad de Talca tiene su origen como tributaria de dos organismos estatales: la Sede de la Universidad Técnica del Estado y el Colegio Universitario de Talca de la Universidad de Chile, los que surgen en un contexto histórico marcado por planes de modernización y descentralización política. Estos veían en el fomento de la educación superior una herramienta imprescindible para formar una ciudadanía democrática y moderna, según explica el programa del partido político en el Gobierno, así como los textos de Jorge Ahumada, Aníbal Pinto y otros pensadores de la época ${ }^{11}$.

Esos esfuerzos centrales recibieron, a su vez, el apoyo de miembros de la sociedad local que lograron convencer a las autoridades nacionales de la necesidad de instalar en la ciudad capital de la Región del Maule, en el centro del Valle Central, región que poseía una rica historia republicana, una identidad que traspasaba sus propios límites geográficos y que se encontraba en una distancia equidistante entre Santiago y Concepción, ambas ciudades con universidades a las que debían recurrir los hijos de la región para continuar estudios superiores. En estas circunstancias parecía evidente la creación de un Centro de Educación Superior en Talca.

En este esfuerzo se encuentran miembros de la élite local como el diputado liberal Guillermo Donoso Vergara y el empresario Jorge Plaza Cruz, más algunos profesionales técnicos, médicos veterinarios, profesores cercanos a escuelas de Artes y Oficios, y un sector de la comunidad italiana. Estos esfuerzos convencieron a muchos que había llegado el momento de poseer una Universidad en su región. Uso el término poseer, pues este grupo se sentirá a lo largo de la historia y particularmente después del año 1973, como "dueños" de la institución, más allá de las decisiones de los propios académicos.

11. Para un análisis de la obra de Jorge Ahumada, véase Pinedo, J., "Lo que estaba en el ambiente. Una lectura de La crisis integral de Chile, Jorge Ahumada, y su relación con el pensamiento de los años 60 en Chile", en Revista Universum, No 18, Universidad de Talca, 2003, págs. 147-172. 
Paralelo a las acciones tendientes a la creación de la sede de la Universidad Técnica del Estado, se efectuaban gestiones para la creación de un colegio regional dependiente de la Universidad de Chile, a través del rector Juan Gómez Millas. Para ello se constituyó un comité denominado "Pro-Colegios Regionales Universitarios en Talca", el que tuvo como misión reunir, por medio de una erogación pública de la comunidad, la suma de cien millones de escudos. Este monto era el aporte que condicionaba la rectoría de la Universidad de Chile a la petición que se formulara sobre la fundación del Colegio Universitario de Talca.

La comunidad talquina simbolizó esta cruzada económica con un "Escudómetro", ubicado en la Plaza de Armas, y en el cual se indicaba de modo gráfico el progreso de la recaudación.

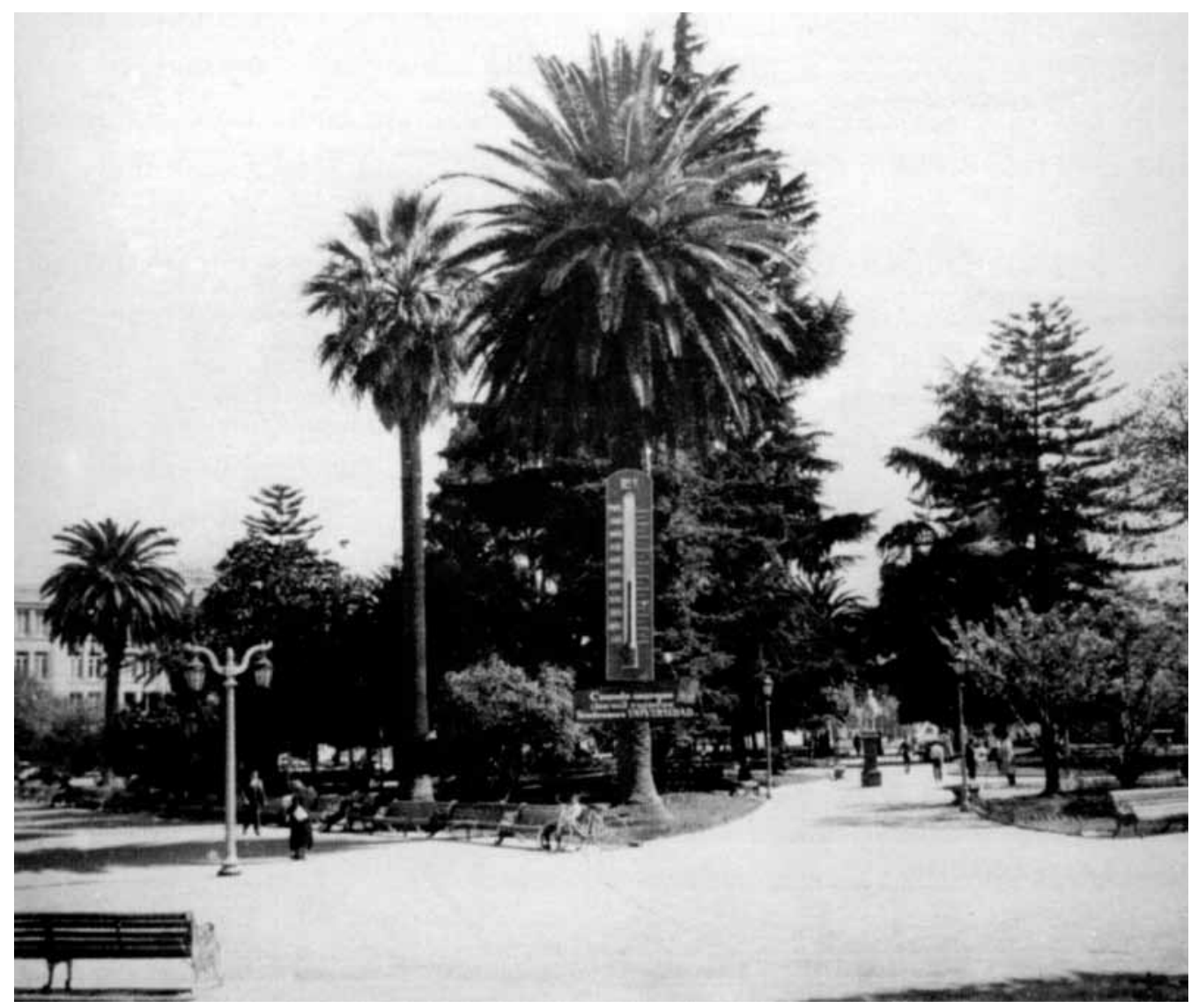

Escudómetro instalado en la Plaza de Armas de Talca para recaudar los fondos necesarios para instalar una Sede de la Universidad de Chile en Talca, 1962. 


\section{Donación}

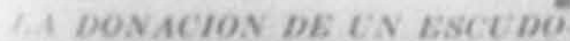
HAHA BH. COLEGIO INATERSI I INIO DE TALCA. DE UN PBN. AIONADO DEL. SERVICIO IIE SE. WI HO SOCTAK. MOWHST $A$ Y SEN WILA EN SI CONTENIIUI TIENE WAA HONHA EXPRESION $U E$ SAVTIIM) IIUMANG - Los perio. distas. duanto tenemos ef deber de 4 rir la cerdad y enfrentar los meis crocos acomlecimientos. en bencileio

\section{enaltece}

broma. жupisegs yue. den Oleguria. 18 ne 72 aniwa de cdad y coto. couna ut roble Ba impuercote dexde gure fundo la Caja del Segure Obligalo Av eu 1925; cmpezdudolo a hacer. cuando trabajaba en tas salitreras qunaba seis pesos diarios Lo hiso rn Ia Ofieina Filomena. de don Paserue Barburizza También. estuco en Pro ra y Bolicia Jubitó como contrafle ta en ciñas Es casado. con una pa

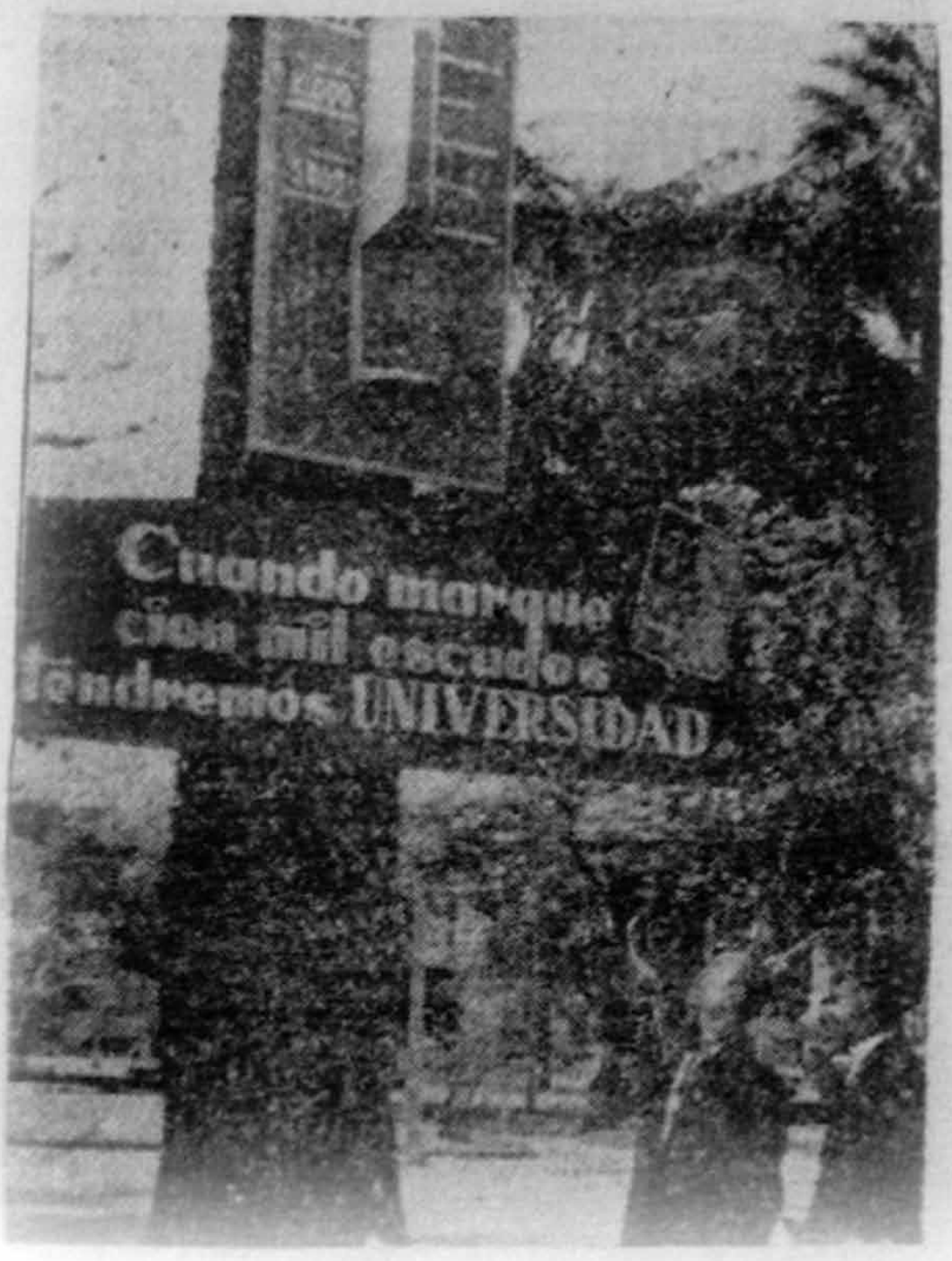

Recorte del Diario La Mañana de Talca. 27 de noviembre de 1962. 
Talca, con el propósito de forjar su propio destino universitario, logró reunir el dinero requerido, cuyo monto estaba destinado a la construcción de la futura Casa de Estudios Superiores.

Sin embargo, y pese a este esfuerzo mancomunado de la ciudadanía, no se contaba con el monto de dinero que permitiera la compra de terrenos, situación que fue salvada gracias a la intervención del matrimonio formado por Romeo Castagnini y Livia Roncagliolo, quienes donaron a la Universidad de Chile una faja de terreno en la zona de Lircay, donde ahora funciona el campus del mismo nombre de la Universidad de Talca.

El comité encargado de toda la gestión fue presidido por el abogado Aurelio Fernández e integrado por el doctor Enrique Mercadal Paccaud; el abogado Mario Villalobos Cruz; el empresario Miguel Calaf Rocosa; el alcalde de la Municipalidad de Talca, Galo Lavín Pradeñas; el Monseñor Manuel Larraín Errázuriz, Obispo de Talca; el presidente de la Asociación Agrícola Central, Enrique Cortés Donoso; el diputado Guillermo Donoso Vergara; el director del Diario La Mañana, Juan C. Bravo Ramos; Ricardo Burgos y Esteban Pérez, por la Cámara de Comercio; Samuel Espinoza de la Asociación de Comerciantes Minoristas y Raúl Lara Sepúlveda representó al Colegio de Periodistas, entre otras personalidades ${ }^{12}$.

De este modo, el 25 de noviembre de 1962, con la asistencia del ministro de Educación Pública de ese entonces, Patricio Barros Alemparte; el rector de la Universidad de Chile, Juan Gómez Millas; el intendente de Talca, José Escobar Puccio; la cesionaria de los terrenos Livia Roncagliolo y otras altas personalidades, se procedió a la solemne ceremonia de colocación de la primera piedra.

El pergamino que quedó incrustado en la piedra reza lo siguiente: "En Talca, a 25 de noviembre de 1962, a las 12:00 Horas se procedió a la bendición y colocación de la primera piedra del edificio de los Colegios Regionales en Talca, en presencia del Señor Ministro de Educación Don Patricio Barros Alemparte, del Señor Rector de la Universidad de Chile Juan Gómez Millas, del Señor Alcalde de Talca, Don Galo Lavín Pradeñas, de Monseñor Ernesto Rivera Reyes y autoridades de la ciudad, que para mayor solemnidad del acto y constancia del mismo, abajo firman".

Así, primero se estableció la Universidad Técnica del Estado en Talca, y más tarde la sede de la Universidad de Chile que se levantará el 26 de octubre de $1964^{13}$ con la creación de la Escuela Universitaria de Talca, cuyo texto de origen dice lo siguiente: "Créase la Escuela Universitaria de Talca dependiente de la Universidad de Chile sobre la base de los actuales cursos universitarios que funcionan en esa ciudad"14.

El año 1962 constituye para la ciudad de Talca un hito importante de su historia, al darse el primer paso de lo que sería su futuro universitario. Quedó, asimismo, en la más completa convicción del rector de la Universidad de Chile de la época, Juan Gómez Millas, que había que iniciar a la brevedad posible la construcción de la futura Universidad del Centro, que favorecería a la extensa zona comprendida entre Santiago y Concepción.

12. AAVV., Universidad de Talca, Veinte Años de Historia, Talca, 2001.

13. Coincidiendo con la fecha de fundación de la futura Universidad de Talca, un 26 de octubre de 1981.

14. AAVV., Universidad de Talca, Veinte Años de Historia, Talca, 2001. 
De este modo, Talca contaba en 1962 con dos sedes universitarias, la de Chile y la Técnica del Estado, que ya había comenzado sus labores en la ciudad.

La lógica adoptada para la implementación de los cursos fue la de aceptar lo que ofrecía académicamente la ciudad a la demanda de los futuros alumnos, de tal modo, si existía un número destacado de profesores de matemáticas, se dictaban esas áreas, si de castellano, igualmente. Así, se abrieron un número importante de pedagogías, carreras de la salud, contabilidad, etc.

Es decir, se creció en tres direcciones: desde lo que brindaba la región, desde las demandas locales y según las directrices centrales. Tal vez, este modus operandi fue similar en todas las regiones donde se extendió la Universidad de Chile, que vio en estas una oportunidad de crecimiento y de aplicación de políticas educacionales en el resto del país.

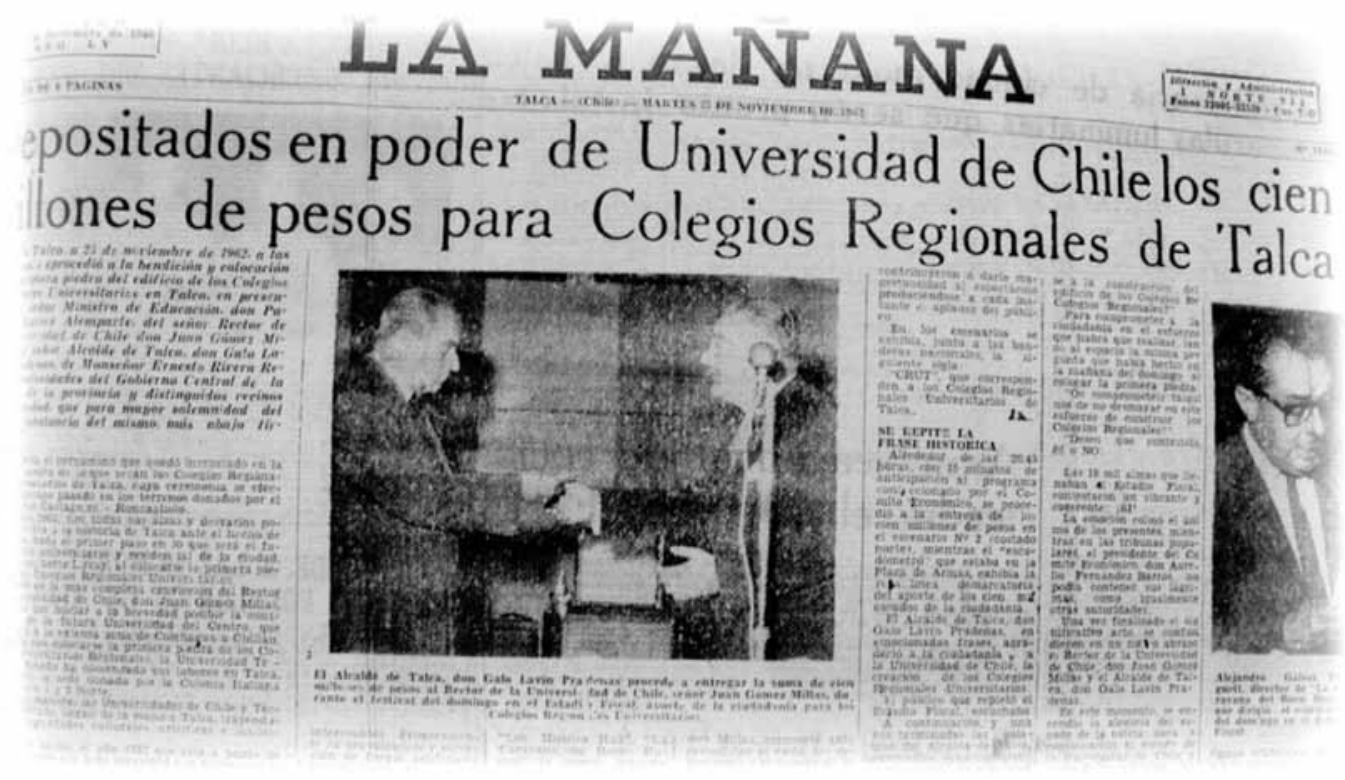

Colocación de la Primera piedra de los Colegios regionales en Talca, 25 noviembre 1962.

El apoyo de la comunidad fue muy importante, y son las decisiones comunitarias, en buena medida, las que sostienen estas acciones. Este apoyo tuvo sus pros y sus contras, pues, por una parte, se trata del compromiso de los habitantes de una región que desde el comienzo siente suya a las nuevas instituciones universitarias; por otra, la presencia de esta comunidad se expresa a través de grupos sociales representados por líderes de provincia. Estos son pequeños propietarios, empresarios de origen extranjero, profesionales y técnicos, que serán los que impongan su visión de lo que deberán ser las nuevas universidades: instituciones concebidas como instrumentos de desarrollo de la industria, del agro, de la economía y de la movilidad social. No se trata de universidades para pensar sobre los grandes temas de la humanidad o la cultura. 
En esta lista de líderes hay algunas "excepciones" más cercanas a una creación de una Universidad más compleja, como el arquitecto Euclides Guzmán Álvarez, que fue el primer director de la sede de la Universidad de Chile en Talca, la cual organizó y dirigió durante más de cuatro años entre 1962 y 1967, en tanto que la Subdirectora fue Alicia Gebhard, que lo sucedió en su cargo ${ }^{15}$. En 1966 el plantel universitario contaba con 16 carreras, 32 cursos y 880 estudiantes, lo que prueba el éxito de su gestión y el buen funcionamiento de los centros regionales.

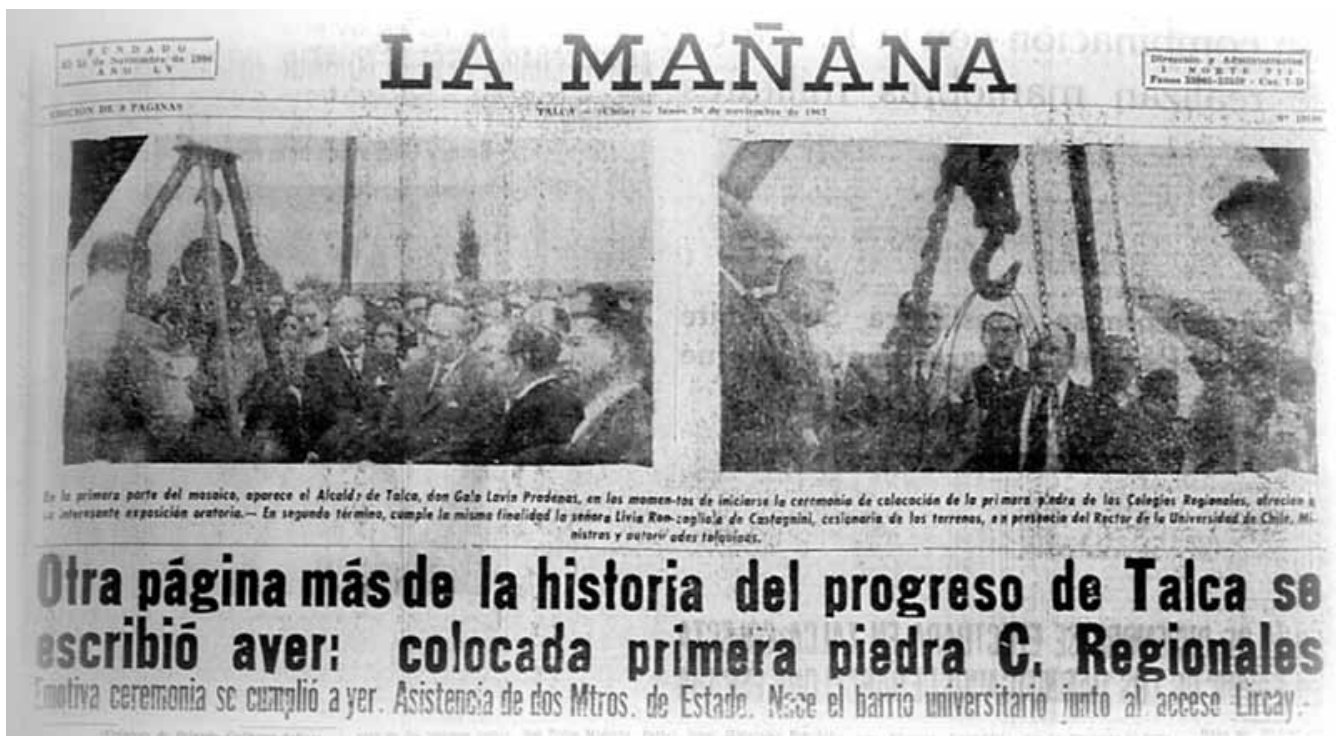

Diario La Mañana, 26 de noviembre de 1962.

Administrativamente, las sedes dependían de las casas centrales en Santiago. Su cuadro directivo estaba conformado por un vicerrector de sede (que reemplazó al director del colegio regional), el Secretario General, el director académico y los directores de departamentos académicos. Las decisiones de este equipo de gestión se concentraban, básicamente, en aspectos administrativos y académicos, estando radicadas las disposiciones legales administrativas en las casas centrales de Santiago, aunque académicamente eran más independientes.

Así, las dos universidades tradicionales, públicas y estatales, la Universidad de Chile y la Universidad Técnica del Estado, se extendieron a casi todo el territorio nacional, a través de sedes o campus regionales, lo que les permitió una mayor presencia en el país

15. "El 4 de julio de 1969 la Municipalidad de Talca lo distinguió con la medalla de oro, en reconocimiento de la labor realizada en la sede la ciudad de la Universidad de Chile, en una sesión solemne presidida por el alcalde, regidores, el obispo de la diócesis y personeros de la Fundación Abate Juan Ignacio Molina". Véase http: //www.fau.uchile.cl/noticias/83626/a-los-95-anos-fallecio-el-destacado-arquitectoeuclides-guzman-alvarez. 
y un aumento importante del número de alumnos en sus aulas. Esta conciencia de una Universidad "nacional", no solo por el nombre que porta, sino porque se extiende a lo largo de la geografía nacional, se fortalecerá a partir del proceso de Reforma Universitaria de los años 1967 y $1970^{16}$, que como se ha indicado, se inició bajo el Gobierno del presidente Frei.

Este ímpetu nacional le permitirá a ambas universidades tradicionales diversificar su oferta académica, crear nuevas facultades y carreras y, sobre todo, un nuevo contacto con la variada realidad regional, influyendo incluso a algunas de las universidades privadas, especialmente a la Universidad Católica que también se expande a regiones, buscando ese mismo contacto con la variedad regional.

En los primeros años de la década de los 70, las sedes regionales de las Universidades de Chile y Técnica del Estado experimentaron progresos notables. La primera logró destacarse en la formación de profesionales en las áreas de la salud y la educación; la Técnica, por su lado, logro lo suyo en las área tecnológicas, de economía y de administración. Gran parte de los profesionales que hoy se desempeñan en el ámbito educacional, en el ámbito de la salud y la administración pública y privada, se formaron en estas sedes universitarias.

Ambas instituciones le fueron otorgando un carácter universitario a la ciudad al crear espacios de reflexión y cultura. Desarrollaron programas de exposiciones de arte y conferencias. Junto a ello, la economía de la ciudad se vio beneficiada por la presencia de una gran cantidad de estudiantes universitarios, lo que originó nuevas posibilidades económicas en el transporte, la alimentación y el pequeño negocio de las pensiones o residencias universitarias ${ }^{17}$.

\section{SISTEMA UNIVERSITARIO Y GOBIERNO MILITAR}

El Gobierno militar afectó profundamente la administración de las sedes regionales. En principio, se mantuvo la estructura de Vicerrectores designados pero reemplazados ahora por figuras sin tradición universitaria, pero sí con mucha adhesión al Gobierno militar, lo que era casi su única garantía de mantenerse en el cargo. Así fue el caso de la sede de la Universidad de Chile, con Pedro Félix de Aguirre, que impuso una administración de sospecha y represión y que en ocasiones obligaba a los académicos a marchar por las calles de la ciudad manifestando su adhesión al Gobierno de Pinochet. El que no lo hacía era expulsado de la Universidad. Pedro Félix de Aguirre venía de dirigir la Universidad Técnica del Estado y fue trasladado en 1976 a la sede de la Universidad de Chile.

16. Véase Núñez, I., "La descentralización y las Reformas Educacionales, 1940-1973", en PIIE Serie Histórica, No 2, cap. IV, Santiago, 1990.

17. Al final de la década de los 70 habían egresado y se habían titulado, en ambas sedes universitarias, una cifra cercana a los 5.000 profesionales en las distintas carreras de pregrado. Véase http: //historia.utalca. $\mathrm{cl} / \mathrm{html} /$ director.html 
El nuevo vicerrector era muy autoritario, marcó a la sede, y tal vez a la futura Universidad, para siempre como una organización disciplinaria: Aguirre usó su cercanía con Pinochet para administrar con mano muy dura a la sede, incluso en contra de aquellos que apoyaban tibiamente al Gobierno militar. Más tarde se hizo nombrar decano de la Facultad de Artes de la Universidad de Chile en Santiago, con varias situaciones rocambolescas que se mantienen vivas entre los testigos de la época ${ }^{18}$.

Más tarde, la reforma del sistema de educación superior impuesto por el grupo de civiles neoliberales que apoyaban a los militares, significó que las antiguas sedes regionales de la Universidad de Chile y Técnica del Estado, distribuidas a lo largo del país, dieran origen a universidades regionales. Lo anterior, visto históricamente, no era más que un paso en relación con la idea original de crear sedes en las regiones que en aquel momento, inicios de los año 60, no se podrían haber creado. Podemos considerar al concepto "sede" como una semilla que llevaría, de una forma u otra, a traducir el deseo de autonomía y en la necesidad de fundar universidades independientes, lo que se materializó a partir de la década de los 80, momento en que se impuso la Reforma de 1981 de forma autoritaria y que significó el desmantelamiento de la Universidad de Chile.

El Gobierno militar, que subió al poder en 1973, nunca tuvo una buena relación con las universidades y menos con la de Chile en particular, a la que acusaba de ser estatal y de ocultar en su interior a pensadores y estudiantes opuestos al Gobierno. Hay muchos testimonios de que siempre prefirió a la Universidad Católica, de donde obtuvo mayoritariamente sus ministros y funcionarios.

En este contexto, resistió mal la existencia de la Universidad de Chile y preparó un plan para su modificación estructural que se implementó a comienzos de los años 80 y que buscaba dividirla para hacerle perder parte de su patrimonio y disminuir su importancia nacional. Es decir, un plan que iba en sentido contrario a lo propuesto en la reforma del tiempo de Frei Montalva. Una "contrarreforma" para las universidades estatales, que se denominó "La Reforma de la Educación Superior", implementada en 1981 y que se constituyó en la mayor modificación que han sufrido las universidades chilenas a lo largo de la historia y que permitió la creación de universidades regionales, producto de la fusión de las antiguas sedes, así como el surgimiento de universidades privadas.

En el caso de Talca, el Gobierno optó por la fusión de las sedes de la Universidad de Chile y de la Universidad Técnica del Estado dando paso a la creación del Instituto Profesional de Talca, que se creó el 10 de marzo de 1981, nombrándose como su primer rector al Coronel del Ejército e Ingeniero Militar, Manuel Toso Giudice, quien hasta esa fecha se desempeñaba como vicerrector de la sede de la Universidad Técnica del Estado. La decisión fue tomada en Santiago sin consultarles en ningún momento a los académicos $^{19}$.

18. Darío Oses, en su novela Machos tristes (1991), reproduce a este Vicerrector como personaje literario.

19. Algunos llegaron a decir que en Talca se estableció un Instituto Profesional y no una Universidad debido a la mala disposición del gobernante que nunca pensó a Talca como una ciudad leal y a su favor. 
La Reforma de 1981 propuso tres tipos de centros de educación superior: las universidades, los institutos profesionales y los Centros de Formación Técnica (CFT). Para las primeras se reservaron doce carreras consideradas (arbitrariamente) de prestigio social y académico. Los institutos profesionales fueron definidos como centros docentes con carreras de cuatro o cinco años de duración, mientras que los Centros de Formación Técnica ofrecían carreras cortas, de no más de dos años de duración.

La reforma de 1981, como hemos dicho, modificó el sistema de sedes regionales, transformándolas en una primera etapa en institutos profesionales y algunos otros, directamente en universidades, quedando el sistema universitario público de la siguiente forma: las sedes que tenía la Universidad de Chile en Arica e Iquique, en unión con la sede de la Universidad Técnica del Estado, pasaron a convertirse en institutos profesionales y, posteriormente, en la Universidad de Tarapacá y Arturo Prat, respectivamente.

Las sedes en Antofagasta de la Universidad de Chile y Técnica del Estado se fusionan en la Universidad de Antofagasta; mientras que la sede Copiapó de la Universidad Técnica se transforma en el Instituto Profesional de Copiapó y, más tarde, en la Universidad de Atacama. La fusión de las sedes de la Universidad de Chile y Técnica dieron origen a la Universidad de La Serena; la sede en Valparaíso de la Universidad de Chile se transforma en Universidad del mismo nombre; el Instituto Pedagógico de Valparaíso, en Academia de Ciencias Pedagógicas de Valparaíso y, más tarde, en la Universidad de Playa Ancha; la propia Universidad de Chile en Santiago (considerada en ocasiones como una sede más), se transforma en la Universidad de Chile con dos derivados, el Instituto Profesional de Santiago, que posteriormente da origen a la Universidad Tecnológica Metropolitana, y el Instituto Pedagógico de Santiago en la Academia de Ciencias Pedagógicas de Santiago y luego, en la Universidad Metropolitana de Ciencias de la Educación. Las sedes de la Universidad de Chile y Técnica en Talca, Chillán y Osorno dan origen a Institutos Profesional con los nombres de las ciudades y, más tarde, a las universidades de Talca, Bío-Bío y Los Lagos. En cambio, las sedes en Temuco dieron paso directamente a la Universidad de la Frontera (¿frontera con quién?) y la sede de la Universidad Técnica en Punta Arenas en el Instituto Profesional de Magallanes y, más tarde, en la Universidad de Magallanes.

Las sedes regionales pasaron a constituirse en nuevas universidades públicas, denominadas "derivadas", por ser herederas de las Universidades de Chile y Técnica del Estado. Esta última, a su vez, modificó su nombre por el de "Universidad de Santiago de Chile".

Como es fácil suponer, en todos estos casos la pérdida patrimonial de las universidades matrices fue enorme.

El Instituto Profesional de Talca alcanzó a existir alrededor de siete meses. Este período significó, en la práctica, unir a las dos sedes universitarias que lo habían originado, con proyectos, cultura y tradición diferentes. Y hay que reconocer el esfuerzo que significó unir dos ex sedes, con rasgos y estilos diferentes, estableciendo equivalencias entre las carreras, adaptando modos de gestión y reglamentación de la vida estudiantil, académica y administrativa. Al crearse el Instituto Profesional de Talca, fue reemplazado 
por el coronel de ejército Manuel Tosso que venía de dirigir la sede de la Técnica del Estado y que gobernó evitando los excesos del vicerrector anterior, el Sr. Aguirre.

Por otro lado, la decisión de crear un instituto profesional y no una Universidad autónoma no era lo que esperaba la comunidad, que vio frustrado el permanente sueño de contar con una Universidad. Pero como no era el momento en que se podía protestar las decisiones del Gobierno, la medida no evitó la gran frustración que produjo en la comunidad de Talca y la región. No hubo protestas pero sí muchos rumores críticos, los que llevaron a que las autoridades del nuevo instituto profesional, encabezadas por el rector Tosso, se dedicaran a una doble tarea: la de organizar al nuevo instituto y, por otra, a redactar un proyecto que lo transformara en Universidad. Para tal efecto se constituyó una comisión de autoridades, académicos designados, además de algunos profesionales de la zona, los que elaboraron un "Estudio para la creación de la Universidad Central de Chile, Juan Ignacio Molina", título que no prosperó.

De esta manera, la década de los 80 se constituyó en una etapa fundacional, durante la cual se definieron algunos criterios para la organización de la futura Universidad que se pudiera presentar como digna de ese título.

Finalmente, el 26 de octubre de 1981 fue publicado en el Diario Oficial el Decreto Ley 36 que notificó la creación de la Universidad de Talca, definida como una institución de educación superior, independiente y autónoma con personalidad jurídica propia.

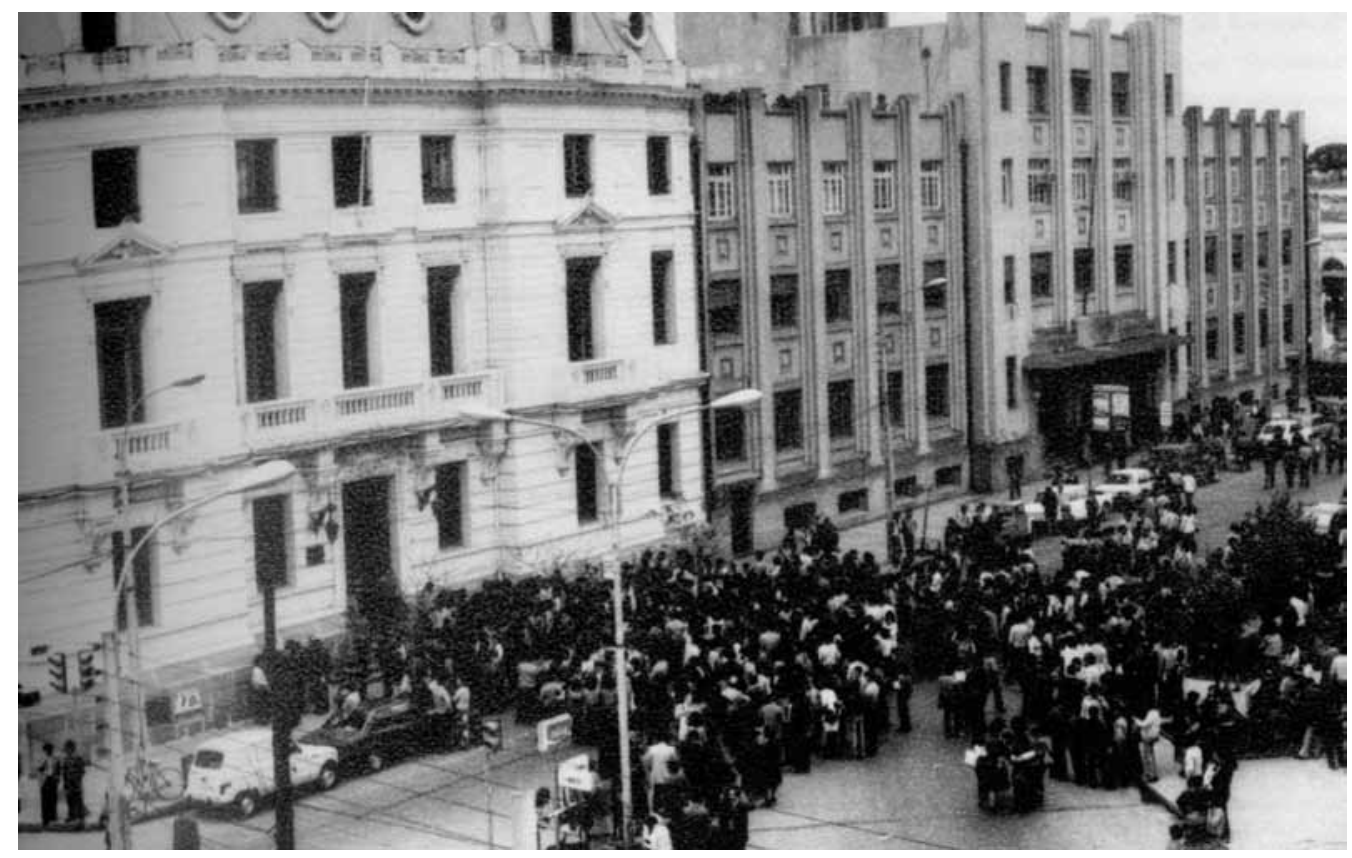

Celebración en Plaza de Armas de Talca, frente a la Intendencia Regional, con motivo de la creación de la Universidad de Talca, 26 de octubre de 1981. 
Los estatutos de las universidades estatales fueron dictados mediante Decretos con Fuerza de Ley durante los años 80. El de la Universidad de Chile fue dictado por D.F.L. $\mathrm{N}^{\circ} 153$ de 1981 y el de la Universidad de Talca, el Decreto Ley No 36 del 26 de octubre del mismo año ${ }^{20}$.

La creación de la Universidad de Talca fue muy bien recibida por la población en general, que vio cumplido el sueño de poseer una Universidad en la ciudad.

Más allá de la pérdida material para la Universidad de Chile, la creación de la Universidad de Talca produjo una renovación interna, tanto en la movilidad académica como en su progresiva democratización, pero, sobre todo, implicó el surgimiento de nuevas oportunidades de desarrollo, de creación de un perfil propio para asegurar su instalación en el competitivo escenario que se avecinaba. Los desafíos ahora consistían en crear y mantener un alto nivel académico que fuera reconocible por el mundo universitario nacional e internacional, así como por la recuperación de la democracia en la gestión interna. No solo estaba el tema de darse una estructura de administración y desarrollar una cultura académica generada a partir de una reflexión propia, sino que, también, definir líneas de desarrollo, establecer vínculos con la comunidad externa, regional y nacional, y determinar los sustentos ideológicos para las actividades de docencia, investigación y extensión. Este momento fundacional estuvo imperado por estas definiciones y por la necesidad de establecer un proyecto de calidad, en un contexto de restricciones presupuestarias, normalización progresiva de la participación y decisiones de los académicos y nuevos escenarios más abiertos a la competencia.

Lo más importante fue que no se podía esperar decisiones desde el centro, sino que se debía crear el futuro con imaginación propia.

El camino recorrido desde entonces ha sido fructífero, tanto en la creación de carreras y facultades como en el crecimiento de sedes en Curicó, Linares, Santa Cruz en Colchagua y Santiago, cada una de ellas con misiones específicas arraigadas en su entorno particular.

En términos generales, se puede señalar que la Universidad de Talca se inició como una institución básicamente docente con un cuerpo académico que no tenía la formación de postgrado necesaria como para desarrollar tareas de investigación de calidad nacional e internacional, aspectos que se han ido alcanzando de manera rápida, lo que ha permitido su destacada ubicación y, desde hace mucho tiempo, entre las diez primeras del país. Decimos esto justo en el momento en que cumple 31 años de vida independiente.

20. Véase Núñez, I., "Historia reciente de la educación chilena", julio de 1997, en http: //historiadelaeducacionenchile.bligoo.cl/media/users/13/699601/files/92631/historia_de_la_educacion_chilena_2.pdf 